A case of Gemella morbillorum endocarditis

Sir,

We report a case of Gemella morbillorum endocarditis. A 75-year-old man was admitted to hospital for investigation of weight loss and lassitude of two months duration. He had a history of rhematic fever as a child. Examination revealed a temperature of $38.5^{\circ} \mathrm{C}$, mild ankle oedema and a pansystolic murmur radiating to the axilla. There were no other signs of endocarditis. He had a haemoglobin of $9.3 \mathrm{~g} / \mathrm{dl}$, white cell count of $9.7 \times 10^{9} / 1$ and an erythrocyte sedimentation rate of $48 \mathrm{~mm} / \mathrm{h}$. An echocardiogram revealed mitral valve vegetations with regurgitation. $A$ diagnosis of endocarditis was made, three sets of blood cultures were taken and he was started on benzylpenicillin $1.2 \mathrm{~g}$ iv 4 hourly and gentamicin $80 \mathrm{mg}$ iv 12 hourly. His temperature returned to normal after 24 hours. At 48 hours all three sets of blood cultures grew $G$ morbillorum (confirmed by the Streptococcal Reference Laboratory). The organism proved sensitive to penicillin on disc testing but failed to survive subculture for further sensitivity studies. After 24 days of benzylpenicillin a bright red maculopapular rash appeared over the patient's trunk and legs and a leucopenia of $1.8 \times 10^{9} / 1$ was noted. This was consistent with an allergic reaction and treatment was stopped. His temperature rose to $39^{\circ} \mathrm{C}$ within 48 hours. Further cultures were taken and he was given teicoplanin. After the first dose of this he became dizzy, wheezy and breathless. His blood pressure fell to $90 / 60 \mathrm{mmHg}$. $\mathrm{He}$ was given iv hydrocortisone, chlorpheniramine and salbutamol. In view of his reaction to teicoplanin we decided not to risk treatment with vancomycin and opted instead for rifampicin $600 \mathrm{mg}$ orally 12 hourly and erythromycin $500 \mathrm{mg}$ orally 6 hourly. The organism had appeared sensitive to both these drugs on disc testing. The patient received a total of four weeks treatment and made a good recovery. He remains well six months later.

$G$ morbillorum is closely related to the 'viridans' type streptococci and is considered a member of the normal upper respiratory tract flora. Infections caused by this organism seem to resemble those caused by viridans streptococci. Bacteraemia and endocarditis seem to predominate ${ }^{1}$ although meningitis and septic arthritis ${ }^{3}$ have been reported. Osman $^{4}$ reports five cases of $G$ morbillorum endocarditis. We report a sixth. Therapy with penicillin and gentamicin is the treatment of choice as most isolates show synergy although tolerance has been demonstrated. ${ }^{5}$ Coto $^{6}$ presents a case in which penicillin plus rifampicin was successful after penicillin plus amikacin had failed. Glycopeptide therapy is recommended in those allergic to betalactams. Since our patient could not tolerate these, a combination of rifampicin plus erythromycin was selected and success achieved. Peard ${ }^{7}$ reports one case where this combination has been used successfully against Staphylococcus aureus endocarditis in which good synergy was demonstrated. We believe this combination is unique in the treatment of 'viridans' type streptococcal endocarditis.

MJ MARTIN

DA WRIGHT

ARR JONES

Department of Microbiology, Worthing Hospital,

West Sussex BN11 2DH, UK

\section{Learning points}

- Gemella morbillorum is closely related to the streptococci and is a rare cause of endocarditis

- penicillin plus an aminoglycoside is the treatment of choice

- rifampicin plus erythromycin is an alternative combination in penicillin-allergic patients

1 Wood CA. Infections caused by Gemella morbillorum. Lancet 1993; 342: 560.

2 Debast SB, Koot R, Meis JFGM. Infections caused by Gemella morbillorum. Lancet 1993; 342: 560 .

3 von Essen R, Ikavalko M, Forsblom B. Isolation of Gemella morbillorum from joint fluid. Lancet of Gemella morbilh

4 Osman Y, Wood CA. Endovascular infection and septic arthritis caused by Gemella morbillorum. Diagn Microbiol Infect Dis 1993; 16: $131-4$

5 Maxwell S. Endocarditis due to Streptococcus morbillorum. F Infect 1989; 18: 67-72.

6 Coto H, Berk SL. Endocarditis caused by Streptococcus morbillorum. Am $\mathcal{f}$ Med Sci 1984; 287(3): 54-8.

7 Peard MC, Fleck DG, Garrod LP, Waterworth PM. Combined rifampicin and erythromycin 410-11.

Gemella haemolysans prosthetic valve endocarditis

Sir,

We report a case of prosthetic valve endocarditis caused by Gemella haemolysans in a 34-year-old man who has had four cardiac operations including valve replacement for complex heart disease. We believe this to be the first reported case in the UK.

A 34-year-old man presented with a short history of myalgia, fever, and general malaise. He recognised these symptoms as possibly being endocarditis, having had four previous episodes. The only positive findings on examination were three sites of asymptomatic dental sepsis. Transoesophageal echocardiography showed a lesion on his prosthetic aortic valve suggestive of a vegetation. $G$ haemolysans was isolated from several blood cultures. G haemolysans is sensitive to penicillin and synergy with gentamicin has been demonstrated. ${ }^{1}$ This option was not open to us as, three years previously, the patient had profound neutropenia, including bone marrow eosinophilia, during another episode of endocarditis treated by benzylpenicillin and gentamicin. The neutropenia resolved on withdrawal of benzylpenicillin. Based on blood culture sensitivities treatment was begun with intravenous cefuroxime and tobramycin; the latter was stopped after 14 days and oral ciprofloxacin substituted. After a further five days the cefuroxime was stopped because the patient developed a profound neutropenia, and oral erythromycin started. Marrow examination revealed a decreased number of myeloid cells, with very little development beyond the myelocyte stage, and an increased number of eosinophils. Erythroid cells and megakaryocytes were normal. The neutrophil count recovered spontaneously over the next four days.

Six cases of endocarditis due to $G$ haemolysans have been reported since $1982,{ }^{2}$ but only one case of prosthetic valve endocarditis. The recommended treatment is a combinaP BLOOMFIELD

Department of Medicine P ROSS

Department of Medical Microbiology, Royal Infirmary of Edinburgh, Edinburgh EH3 $9 Y W, U K$

tion of penicillin and aminoglycoside. ${ }^{1,2}$ In

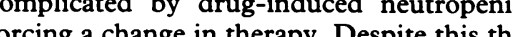
patient made a good recovery and remains well with no deterioration in his prosthetic valve function.

Correspondence to Dr L Samuel, Clinical Oncology Road, Edinburgh EH4 2XU, UK

1 Buu-Hoi A, Sapoetra A, Branger C, Acar JF Antimicrobial susceptibility of Gemelle haemolysans isolated from patients with haemolysans isolated from patients with subacute endocarditis

2 Fresard A, Michel VP, Rueda G, Aubert G, Dorche G, Lucht F. Gemella haemolysans endocarditis. Clin Infect Dis 1993; 16: 586-7.

3 Morea P, Toni M, Bressan M, Stritoni P Prosthetic valve endocarditis by Gemella haemolysans. Infection 1991; 19: 446

\section{Aplastic anaemia or aplastic pancytopaenia?}

Sir,

The term aplastic anaemia is an unsuitable and confusing term extensively used in the medical literature. It is still present in the recent editions of textbooks of internal medicine and haematology, ${ }^{1,2}$ and in most medical journals. ${ }^{3-5}$

The term 'aplastic anaemia' refers to conditions in which a markedly hypocellular bone marrow results in pancytopaenia (anaemia, leukopaenia, and thrombocytopaenia). ${ }^{1}$ However, from a terminological point of view, aplastic anaemia means anaemia due to aplastic bone marrow where this anaemia in the medical literature is termed pure red cell aplasia. So I suggest that aplastic anaemia should be used only when the aplasia involves a selective failure in the production of erythroid elements in the bone marrow causing anaemia and reticulocytopaenia. When panpresent, instead of the classic term aplastic anaemia, the term aplastic pancytopaenia should be used; it is both more descriptive, and more suitable.

MAJED ODEH

Department of Internal Medicine B, Bnai Zion Medical Centre, Haifa, Israel

1 Rappeport JM, Bunn HF. Bone marrow failure: aplastic anemia and other primary bone marrow disorders. In: Isselbacher $\mathrm{KJ}$, Braunwald $\mathrm{E}$, Wilson JD, Martin JB, Fauci AS, Kasper DL, eds. Harrison's principles of internal medicine. 13 th edn, Vol. 2. New York: McGraw-Hill, 1994; pp 1754-7.

2 Adamson JW, Erslev AJ. Aplastic anemia. In: Williams WJ, Beutler E, Erslev AJ, Lichtman MA, eds. Hematology. 4th edn. New York: MA, eds. Hematology. 4th edn.

3 Schubert J, Vogt HG, Skowrnek-Zielinska M, et Schubert J, Vogt HG, Skowrnek-Zielinska M, et sitol-anchoring defect characteristic for paroxysitol-anchoring defect characteristic for paroxysmal nocturnal hemoglobinuria in patients aplastic anemia. Blood 1994; 83: 2323-8.

4 Narayanan MN, Geary CG, Freemont A, Kendra JR. Long-term follow-up of aplas
Br f Haematol 1994; 86: 837-43.

5 Nistica A, Young NS. Gamma-interferon gene expression in the bone marrow of patients with aplastic anemia. Ann Intern Med 1994; 120: 463-9. this case management of the patient was cytopaenia due to bone marrow aplasia is 